\title{
PARTIAL FRACTION DECOMPOSITIONS AND TRIGONOMETRIC SUM IDENTITIES
}

\author{
WENCHANG CHU \\ (Communicated by Carmen C. Chicone)
}

\begin{abstract}
The partial fraction decomposition method is explored to establish several interesting trigonometric function identities, which may have applications to the evaluation of classical multiple hypergeometric series, trigonometric approximation and interpolation.
\end{abstract}

\section{Outline And introduction}

Recently, in an attempt to prove, through the Cauchy residue method, Dougall's theorem (Dougall [6, 1907], see [5] also) on the well-poised bilateral hypergeometric ${ }_{5} \mathrm{H}_{5}$-series, the author found the following trigonometric sum identity:

$$
\begin{aligned}
\frac{\sin a}{\sin b \sin c \sin d \sin e} & =\frac{\cot b \sin (a-2 b)}{\sin (c-b) \sin (d-b) \sin (e-b)} \\
& +\frac{\cot c \sin (a-2 c)}{\sin (b-c) \sin (d-c) \sin (e-c)} \\
& +\frac{\cot d \sin (a-2 d)}{\sin (b-d) \sin (c-d) \sin (e-d)} \\
& +\frac{\cot e \sin (a-2 e)}{\sin (b-e) \sin (c-e) \sin (d-e)}
\end{aligned}
$$

Consider the trigonometric fraction defined by

$$
R(z):=\frac{e^{i z} \cot z \sin (a+2 z)}{\sin (b+z) \sin (c+z) \sin (d+z) \sin (e+z)} .
$$

According to the Euler formulae

$$
\cos z=\frac{e^{i z}+e^{-i z}}{2} \text { and } \sin z=\frac{e^{i z}-e^{-i z}}{2 i}
$$

this rational function is essentially a fraction in $e^{i z}$ with the degree of the numerator polynomial less than that of the denominator polynomial by one. Therefore we can

Received by the editors October 25, 2006.

2000 Mathematics Subject Classification. Primary 42A15; Secondary 65T40.

Key words and phrases. Trigonometric interpolation, trigonometric formulae, partial fraction decomposition. 
decompose it into the following partial fractions:

$$
R(z)=\frac{A}{\sin z}+\frac{B}{\sin (b+z)}+\frac{C}{\sin (c+z)}+\frac{D}{\sin (d+z)}+\frac{E}{\sin (e+z)}
$$

where the coefficients $\{A, B, C, D, E\}$ are determined respectively by

$$
\begin{aligned}
A & =\lim _{z \rightarrow 0} z R(z)=\frac{\sin a}{\sin b \sin c \sin d \sin e}, \\
B & =\lim _{z \rightarrow-b}(b+z) R(z)=\frac{-e^{-b i} \cot b \sin (a-2 b)}{\sin (c-b) \sin (d-b) \sin (e-b)}, \\
C & =\lim _{z \rightarrow-c}(c+z) R(z)=\frac{-e^{-c i} \cot c \sin (a-2 c)}{\sin (b-c) \sin (d-c) \sin (e-c)}, \\
D & =\lim _{z \rightarrow-d}(d+z) R(z)=\frac{-e^{-d i} \cot d \sin (a-2 d)}{\sin (b-d) \sin (c-d) \sin (e-d)}, \\
E & =\lim _{z \rightarrow-e}(e+z) R(z)=\frac{-e^{-e i} \cot e \sin (a-2 e)}{\sin (b-e) \sin (c-e) \sin (d-e)} .
\end{aligned}
$$

Recall that

$$
\sin M i=i \sinh M, \quad \cos M i=\cosh M \quad \text { and } \quad \lim _{M \rightarrow+\infty} \frac{\sinh M}{\cosh M}=1 .
$$

For $M \rightarrow+\infty$, we can check without difficulty the following limiting relations:

$$
\begin{aligned}
\frac{\sin M i}{\sin (\lambda+M i)} & =\frac{i \sinh M}{\sin \lambda \cosh M+i \cos \lambda \sinh M} \rightarrow e^{\lambda i} \\
R(M i) \sin M i & =\frac{e^{-M} \cos M i \sin (a+2 M i)}{\sin (b+M i) \sin (c+M i) \sin (d+M i) \sin (e+M i)} \\
& \approx \frac{2 i \exp \{(b+c+d+e-a) i\}}{e^{M} \sinh M} \rightarrow 0
\end{aligned}
$$

Multiplying across (2) by $\sin z$, then letting $z=M i$ and $M \rightarrow+\infty$, we confirm the trigonometric sum identity displayed in (1).

This trigonometric sum identity is only the tip of the hidden iceberg. In fact, there exist many more trigonometric formulae of the same nature, which will be established by means of partial fraction decomposition method. The main theorems will be shown in the next section through this unified and more accessible approach.

Then the last section will illustrate several interesting examples, including those due to Calogero [2, §2.4.5.3], Gustafson [8,9] and Mohlenkamp-Monzón [11]. As demonstrated in these works just cited, the trigonometric identities presented in this paper may find further application in the evaluation of classical multiple hypergeometric series, trigonometric approximation and interpolation.

\section{PARTial FRACTION DECOMPOSITION}

Consider the following function:

$$
R(z)=\frac{P\left(e^{i z}\right)}{\prod_{k=0}^{n} \sin \left(z-\beta_{k}\right)},
$$

where $P(w)$ is a Laurent polynomial in $w$ consisting of terms $w^{k}$ with $|k| \leq n+1$ and $\left\{\beta_{k}\right\}_{k=0}^{n}$ are $n+1$ distinct complex numbers. 
Noting that

$$
\sin (z-\beta)=\frac{e^{i(z-\beta)}-e^{i(\beta-z)}}{2 i}=\frac{e^{-i(z+\beta)}}{2 i}\left\{e^{2 i z}-e^{2 i \beta}\right\},
$$

we can reformulate $R(z)$ explicitly in $e^{i z}$ as follows:

$$
R(z)=(2 i)^{n+1} e^{\mathcal{B} i} \frac{P\left(e^{i z}\right) e^{(n+1) i z}}{\prod_{k=0}^{n}\left(e^{2 i z}-e^{2 i \beta_{k}}\right)} \quad \text { where } \quad \mathcal{B}=\sum_{k=0}^{n} \beta_{k} .
$$

Denote by $\left[w^{m}\right] P(w)$ the coefficient of $w^{m}$ in $P(w)$. Then we have the following decomposition in partial fractions:

$$
\frac{P(w) w^{n+1}}{\prod_{k=0}^{n}\left(w^{2}-\gamma_{k}^{2}\right)}=\left[w^{n+1}\right] P(w)+\frac{1}{2} \sum_{k=0}^{n} \frac{\gamma_{k}^{n}}{\prod_{\ell \neq k}\left(\gamma_{k}^{2}-\gamma_{\ell}^{2}\right)}\left\{\frac{P\left(\gamma_{k}\right)}{w-\gamma_{k}}+(-1)^{n} \frac{P\left(-\gamma_{k}\right)}{w+\gamma_{k}}\right\}
$$

where $w=e^{i z}$ and $\gamma_{k}=e^{i \beta_{k}}$ for $0 \leq k \leq n$.

According to the parity of $n$ and $P(w)$, we can combine the fractions inside the last braces $\{\cdots\}$ and simplify the result as follows:

$$
\begin{aligned}
& \frac{P(w) w^{n+1}}{\prod_{k=0}^{n}\left(w^{2}-\gamma_{k}^{2}\right)}=\left[w^{n+1}\right] P(w) \\
& + \begin{cases}\sum_{k=0}^{n} \frac{w P\left(\gamma_{k}\right) \gamma_{k}^{n}}{\left(w^{2}-\gamma_{k}^{2}\right) \prod_{\ell \neq k}\left(\gamma_{k}^{2}-\gamma_{\ell}^{2}\right)}, & n \text { - even : } P(w)=P(-w), \\
\sum_{k=0}^{n} \frac{P\left(\gamma_{k}\right) \gamma_{k}^{n+1}}{\left(w^{2}-\gamma_{k}^{2}\right) \prod_{\ell \neq k}\left(\gamma_{k}^{2}-\gamma_{\ell}^{2}\right)}, & n-\operatorname{even~}: P(w)=-P(-w),\end{cases}
\end{aligned}
$$

$\S 2.1 . P(w)$ and $n$ have the same parity. Suppose throughout this subsection that $(-1)^{n} P(-w)=P(w)$, i.e., the natural number $n$ and the Laurent polynomial $P(w)$ have the same parity.

Noting that

$$
\begin{aligned}
w^{2}-\gamma_{k}^{2} & =2 i e^{i\left(z+\beta_{k}\right)} \sin \left(z-\beta_{k}\right), \\
\gamma_{k}^{2}-\gamma_{\ell}^{2} & =2 i e^{i\left(\beta_{k}+\beta_{\ell}\right)} \sin \left(\beta_{k}-\beta_{\ell}\right),
\end{aligned}
$$

we may restate the first case of (4) in terms of a trigonometric sum identity.

Theorem 1 (n-even with $P(w)=P(-w)$ and $n$-odd with $P(w)=-P(-w)$ ). Let $P(w)$ be a Laurent polynomial consisting of terms $w^{k}$ with $|k| \leq n+1$ and $\left\{\beta_{k}\right\}_{k=0}^{n}$ distinct complex numbers with the sum being denoted by $\mathcal{B}=\sum_{k=0}^{n} \beta_{k}$. Then there holds the summation formula:

$$
\sum_{k=0}^{n} \frac{P\left(e^{i \beta_{k}}\right)}{\sin \left(z-\beta_{k}\right) \prod_{\ell \neq k} \sin \left(\beta_{k}-\beta_{\ell}\right)}=\frac{P\left(e^{i z}\right)}{\prod_{k=0}^{n} \sin \left(z-\beta_{k}\right)} .
$$

In particular for $z=0$, this theorem reduces to the following identity.

Corollary 2 ( $n$-even with $P(w)=P(-w)$ and $n$-odd with $P(w)=-P(-w)$ ). Let $P(w)$ be a Laurent polynomial consisting of terms $w^{k}$ with $|k| \leq n+1$ and $\left\{\beta_{k}\right\}_{k=0}^{n}$ 
distinct complex numbers with the sum being denoted by $\mathcal{B}=\sum_{k=0}^{n} \beta_{k}$. Then there holds the summation formula:

$$
\sum_{k=0}^{n} \frac{P\left(e^{i \beta_{k}}\right)}{\sin \beta_{k} \prod_{\ell \neq k} \sin \left(\beta_{k}-\beta_{\ell}\right)}=\frac{(-1)^{n} P(1)}{\prod_{k=0}^{n} \sin \beta_{k}} .
$$

When $P(w)$ is a Laurent polynomial consisting of terms $w^{k}$ with $|k| \leq n$, we may multiply by $e^{i z}$ across the equation displayed in Theorem 1 . Let $z=-M i$ and $M \rightarrow+\infty$ in the resulting equation and then applying the limiting relation

$$
\lim _{\substack{z=-M i \\ M \rightarrow+\infty}} \frac{e^{i z}}{\sin \left(z-\beta_{k}\right)}=\lim _{M \rightarrow+\infty} \frac{2 i e^{M}}{e^{M-i \beta_{k}}-e^{-M+i \beta_{k}}}=2 i e^{i \beta_{k}},
$$

we derive the following trigonometric identity.

Proposition 3 (n-even with $P(w)=P(-w)$ and $n$-odd with $P(w)=-P(-w)$ ). Let $P(w)$ be a Laurent polynomial consisting of terms $w^{k}$ with $|k| \leq n$ and $\left\{\beta_{k}\right\}_{k=0}^{n}$ distinct complex numbers with the sum being denoted by $\mathcal{B}=\sum_{k=0}^{n} \beta_{k}$. Then there holds the summation formula:

$$
\sum_{k=0}^{n} \frac{e^{i \beta_{k}} P\left(e^{i \beta_{k}}\right)}{\prod_{\ell \neq k} \sin \left(\beta_{k}-\beta_{\ell}\right)}=(2 i)^{n} e^{\mathcal{B} i}\left[w^{n}\right] P(w) .
$$

$\S 2.2 . P(w)$ and $n$ have the opposite parity. Suppose throughout this subsection that $(-1)^{n} P(-w)=-P(w)$, i.e., the natural number $n$ and the Laurent polynomial $P(w)$ have the opposite parity.

Similarly, the second case of (4) may be expressed in terms of another trigonometric sum identity.

Theorem 4 (n-even with $P(w)=-P(-w)$ and $n$-odd with $P(w)=P(-w)$ ). Let $P(w)$ be a Laurent polynomial consisting of terms $w^{k}$ with $|k| \leq n+1$ and $\left\{\beta_{k}\right\}_{k=0}^{n}$ distinct complex numbers with the sum being denoted by $\mathcal{B}=\sum_{k=0}^{n} \beta_{k}$. Then there holds the summation formula:

$$
\sum_{k=0}^{n} \frac{e^{i\left(\beta_{k}-z\right)} P\left(e^{i \beta_{k}}\right)}{\sin \left(z-\beta_{k}\right) \prod_{\ell \neq k} \sin \left(\beta_{k}-\beta_{\ell}\right)}=\frac{P\left(e^{i z}\right)}{\prod_{k=0}^{n} \sin \left(z-\beta_{k}\right)}-(2 i)^{n+1} e^{\mathcal{B} i}\left[w^{n+1}\right] P(w) .
$$

In particular for $z=0$, Theorem 4 reduces to the following identity.

Corollary 5 (n-even with $P(w)=-P(-w)$ and $n$-odd with $P(w)=P(-w)$ ). Let $P(w)$ be a Laurent polynomial consisting of terms $w^{k}$ with $|k| \leq n+1$ and $\left\{\beta_{k}\right\}_{k=0}^{n}$ distinct complex numbers with the sum being denoted by $\mathcal{B}=\sum_{k=0}^{n} \beta_{k}$. Then there holds the summation formula:

$$
\sum_{k=0}^{n} \frac{e^{i \beta_{k}} P\left(e^{i \beta_{k}}\right)}{\sin \beta_{k} \prod_{\ell \neq k} \sin \left(\beta_{k}-\beta_{\ell}\right)}=\frac{(-1)^{n} P(1)}{\prod_{k=0}^{n} \sin \beta_{k}}+(2 i)^{n+1} e^{\mathcal{B} i}\left[w^{n+1}\right] P(w) .
$$

Multiplying by $e^{i z}$ across the equation displayed in Theorem 4, we find that the resulting identity is consistent with that displayed in Theorem 1, but with the degree of numerator polynomial being altered.

Analogously, we can derive from Theorem 4 by letting $z=M i$ and $M \rightarrow+\infty$ the following limiting case. 
Proposition 6 (n-even with $P(w)=-P(-w)$ and $n$-odd with $P(w)=P(-w))$. Let $P(w)$ be a Laurent polynomial consisting of terms $w^{k}$ with $|k| \leq n+1$ and $\left\{\beta_{k}\right\}_{k=0}^{n}$ distinct complex numbers with the sum being denoted by $\mathcal{B}=\sum_{k=0}^{n} \beta_{k}$. Then there holds the summation formula:

$$
\sum_{k=0}^{n} \frac{P\left(e^{i \beta_{k}}\right)}{\prod_{\ell \neq k} \sin \left(\beta_{k}-\beta_{\ell}\right)}=(2 i)^{n} e^{\mathcal{B} i}\left[w^{n+1}\right] P(w)+(-2 i)^{n} e^{-\mathcal{B} i}\left[w^{-n-1}\right] P(w) .
$$

\section{EXAMPLES OF TRIGONOMETRIC IDENTITIES}

The general results displayed in the last section imply numerous identities on trigonometric sums, which will be exhibited in this section.

$\S 3.1$. With the trigonometric polynomial $P(w)$ being defined by

$$
P\left(e^{i z}\right)=e^{-i z} \cos \left(z-\beta_{0}\right) \sin (\alpha-2 z),
$$

we have $P(w)=P(-w)$ which follows from

$$
P(w)=\frac{w^{-4}}{4 i} e^{-i\left(\alpha+\beta_{0}\right)}\left(e^{2 i \alpha}-w^{4}\right)\left(w^{2}+e^{2 i \beta_{0}}\right) .
$$

When $n=2 m$ with $m \geq 2$, we get from Proposition 3 the following identity:

$$
\frac{\sin \left(\alpha-2 \beta_{0}\right)}{\prod_{\ell=1}^{2 m} \sin \left(\beta_{\ell}-\beta_{0}\right)}=\sum_{k=1}^{2 m} \frac{\cot \left(\beta_{k}-\beta_{0}\right) \sin \left(\alpha-2 \beta_{k}\right)}{\prod_{\ell \neq k} \sin \left(\beta_{\ell}-\beta_{k}\right)} .
$$

Putting $\beta_{0}=0$ and $m=2$, we reconfirm the identity anticipated in (1).

$\S 3.2$. With the trigonometric polynomial $P(w)$ being defined by

$$
P\left(e^{i z}\right)=\prod_{k=1}^{n} \sin \left(z-\alpha_{k}\right) \quad \text { and } \quad P(w)=\frac{w^{-n}}{(2 i)^{n}} \prod_{k=1}^{n} e^{-i \alpha_{k}}\left(w^{2}-e^{2 i \alpha_{k}}\right),
$$

the relation $P(w)=(-1)^{n} P(-w)$ implies that $P(w)$ has the same parity as $n$. Then Theorem 1 leads us to the following identity due to Gustafson [8, Lemma 2.14]:

$$
\frac{\prod_{j=1}^{n} \sin \left(z-\alpha_{j}\right)}{\prod_{\jmath=0}^{n} \sin \left(z-\beta_{\jmath}\right)}=\sum_{k=0}^{n} \frac{\prod_{j=1}^{n} \sin \left(\alpha_{j}-\beta_{k}\right)}{\sin \left(z-\beta_{k}\right) \prod_{\jmath \neq k} \sin \left(\beta_{\jmath}-\beta_{k}\right)} .
$$

$\S 3.3$. Let the trigonometric polynomial $P(w)$ be defined by

$$
P\left(e^{i z}\right)=\prod_{k=0}^{n} \sin \left(z-\alpha_{k}\right) \quad \text { and } \quad P(w)=\frac{w^{-n-1}}{(2 i)^{n+1}} \prod_{k=0}^{n} e^{-i \alpha_{k}}\left(w^{2}-e^{2 i \alpha_{k}}\right) .
$$

Then $n$ and $P(w)$ have the opposite parity for $P(w)=(-1)^{n+1} P(-w)$. Consequently, we derive from Theorem 4 the following identity:

$$
\sum_{k=0}^{n} \frac{e^{i\left(\beta_{k}-z\right)} \prod_{j=0}^{n} \sin \left(\alpha_{j}-\beta_{k}\right)}{\sin \left(z-\beta_{k}\right) \prod_{\jmath \neq k} \sin \left(\beta_{\jmath}-\beta_{k}\right)}=\exp \left\{i \sum_{k=0}^{n}\left(\beta_{k}-\alpha_{k}\right)\right\}-\frac{\prod_{j=0}^{n} \sin \left(z-\alpha_{j}\right)}{\prod_{\jmath=0}^{n} \sin \left(z-\beta_{\jmath}\right)}
$$

Extracting the real part across the last equation, we get the identity

(8) $\sum_{k=0}^{n} \cot \left(z-\beta_{k}\right) \frac{\prod_{j=0}^{n} \sin \left(\alpha_{j}-\beta_{k}\right)}{\prod_{\jmath \neq k} \sin \left(\beta_{\jmath}-\beta_{k}\right)}=\cos \left\{\sum_{k=0}^{n}\left(\alpha_{k}-\beta_{k}\right)\right\}-\frac{\prod_{j=0}^{n} \sin \left(z-\alpha_{j}\right)}{\prod_{\jmath=0}^{n} \sin \left(z-\beta_{\jmath}\right)}$. 
The imaginary part recovers another trigonometric formula due to Gustafson [9, Lemma 5.10]:

$$
\sum_{k=0}^{n} \frac{\prod_{j=0}^{n} \sin \left(\alpha_{j}-\beta_{k}\right)}{\prod_{\jmath \neq k} \sin \left(\beta_{\jmath}-\beta_{k}\right)}=\sin \left\{\sum_{k=0}^{n}\left(\alpha_{k}-\beta_{k}\right)\right\} .
$$

There exist different proofs for this last identity, for example, the induction proof by Mohlenkamp-Monzón [11, Theorem 2] and the matrix spectrum method due to Calogero $[2, \S 2.4 .5 .3]$ where more identities can also be verified by means of trigonometric interpolation (cf. Kress $[10, \S 8.2])$.

$\S 3.4$. Let $P(w)$ and $\mathcal{P}(w)$ be two trigonometric polynomials defined respectively by

$$
\begin{aligned}
& P\left(e^{i z}\right)=\sin (\alpha+m z) \quad \text { and } \quad P(w)=\frac{w^{-m}}{2 i} e^{i \alpha}\left(w^{2 m}-e^{-2 i \alpha}\right), \\
& \mathcal{P}\left(e^{i z}\right)=\cos (\alpha+m z) \quad \text { and } \quad \mathcal{P}(w)=\frac{w^{-m}}{2} e^{i \alpha}\left(w^{2 m}+e^{-2 i \alpha}\right) .
\end{aligned}
$$

When $m$ and $n$ are two natural numbers with the same parity and $0 \leq m \leq n$, we derive from Theorem 1 the following two trigonometric sum identities:

$$
\begin{aligned}
& \sum_{k=0}^{n} \frac{\sin \left(\alpha+m \beta_{k}\right)}{\sin \left(z-\beta_{k}\right) \prod_{\ell \neq k} \sin \left(\beta_{k}-\beta_{\ell}\right)}=\frac{\sin (\alpha+m z)}{\prod_{k=0}^{n} \sin \left(z-\beta_{k}\right)}, \\
& \sum_{k=0}^{n} \frac{\cos \left(\alpha+m \beta_{k}\right)}{\sin \left(z-\beta_{k}\right) \prod_{\ell \neq k} \sin \left(\beta_{k}-\beta_{\ell}\right)}=\frac{\cos (\alpha+m z)}{\prod_{k=0}^{n} \sin \left(z-\beta_{k}\right)} .
\end{aligned}
$$

Further, Proposition 3 gives us two other summation formulae:

$$
\begin{aligned}
\sum_{k=0}^{n} \frac{e^{i \beta_{k}} \sin \left(\alpha+m \beta_{k}\right)}{\prod_{\ell \neq k} \sin \left(\beta_{k}-\beta_{\ell}\right)} & =(2 i)^{n-1} e^{i(\alpha+\mathcal{B})} \delta_{m, n}, \\
\sum_{k=0}^{n} \frac{e^{i \beta_{k}} \cos \left(\alpha+m \beta_{k}\right)}{\prod_{\ell \neq k} \sin \left(\beta_{k}-\beta_{\ell}\right)} & =(2 i)^{n-1} i e^{i(\alpha+\mathcal{B})} \delta_{m, n},
\end{aligned}
$$

where $\delta_{m, n}$ stands for the usual Kronecker symbol.

$\S 3.5$. Let $P(w)$ and $\mathcal{P}(w)$ be two trigonometric polynomials defined respectively by

$$
\begin{aligned}
& P\left(e^{i z}\right)=\sin (\alpha+m z) \quad \text { and } \quad P(w)=\frac{w^{-m}}{2 i} e^{i \alpha}\left(w^{2 m}-e^{-2 i \alpha}\right), \\
& \mathcal{P}\left(e^{i z}\right)=\cos (\alpha+m z) \quad \text { and } \quad \mathcal{P}(w)=\frac{w^{-m}}{2} e^{i \alpha}\left(w^{2 m}+e^{-2 i \alpha}\right) .
\end{aligned}
$$

When $m$ and $n$ are two natural numbers with different parity and $0 \leq m \leq n$, we derive from Theorem 4 the following two trigonometric sum identities:

$$
\begin{aligned}
& \sum_{k=0}^{n} \frac{e^{i\left(\beta_{k}-z\right)} \sin \left(\alpha+m \beta_{k}\right)}{\sin \left(z-\beta_{k}\right) \prod_{\ell \neq k} \sin \left(\beta_{k}-\beta_{\ell}\right)}=\frac{\sin (\alpha+m z)}{\prod_{k=0}^{n} \sin \left(z-\beta_{k}\right)}, \\
& \sum_{k=0}^{n} \frac{e^{i\left(\beta_{k}-z\right)} \cos \left(\alpha+m \beta_{k}\right)}{\sin \left(z-\beta_{k}\right) \prod_{\ell \neq k} \sin \left(\beta_{k}-\beta_{\ell}\right)}=\frac{\cos (\alpha+m z)}{\prod_{k=0}^{n} \sin \left(z-\beta_{k}\right)} .
\end{aligned}
$$


Their real and imaginary parts yield respectively the following identities:

$$
\begin{aligned}
& \sum_{k=0}^{n} \frac{\cot \left(z-\beta_{k}\right) \sin \left(\alpha+m \beta_{k}\right)}{\prod_{\ell \neq k} \sin \left(\beta_{k}-\beta_{\ell}\right)}=\frac{\sin (\alpha+m z)}{\prod_{k=0}^{n} \sin \left(z-\beta_{k}\right)}, \\
& \sum_{k=0}^{n} \frac{\cot \left(z-\beta_{k}\right) \cos \left(\alpha+m \beta_{k}\right)}{\prod_{\ell \neq k} \sin \left(\beta_{k}-\beta_{\ell}\right)}=\frac{\cos (\alpha+m z)}{\prod_{k=0}^{n} \sin \left(z-\beta_{k}\right)} ; \\
& \sum_{k=0}^{n} \frac{\sin \left(\alpha+m \beta_{k}\right)}{\prod_{\ell \neq k} \sin \left(\beta_{k}-\beta_{\ell}\right)}=\sum_{k=0}^{n} \frac{\cos \left(\alpha+m \beta_{k}\right)}{\prod_{\ell \neq k} \sin \left(\beta_{k}-\beta_{\ell}\right)}=0 .
\end{aligned}
$$

$\S 3.6$. Let $P(w)$ and $\mathcal{P}(w)$ be two trigonometric polynomials defined respectively by

$$
\begin{aligned}
& P\left(e^{i z}\right)=\sin ^{m}(\alpha+z) \quad \text { and } \quad P(w)=\frac{w^{-m}}{(2 i)^{m}} e^{m i \alpha}\left(w^{2}-e^{-2 i \alpha}\right)^{m}, \\
& \mathcal{P}\left(e^{i z}\right)=\cos ^{m}(\alpha+z) \quad \text { and } \quad \mathcal{P}(w)=\frac{w^{-m}}{2^{m}} e^{m i \alpha}\left(w^{2}+e^{-2 i \alpha}\right)^{m} .
\end{aligned}
$$

When $m$ and $n$ are two natural numbers with the same parity and $0 \leq m \leq n$, we derive from Theorem 1 the following two trigonometric sum identities:

$$
\begin{aligned}
& \sum_{k=0}^{n} \frac{\sin ^{m}\left(\alpha+\beta_{k}\right)}{\sin \left(z-\beta_{k}\right) \prod_{\ell \neq k} \sin \left(\beta_{k}-\beta_{\ell}\right)}=\frac{\sin ^{m}(\alpha+z)}{\prod_{k=0}^{n} \sin \left(z-\beta_{k}\right)}, \\
& \sum_{k=0}^{n} \frac{\cos ^{m}\left(\alpha+\beta_{k}\right)}{\sin \left(z-\beta_{k}\right) \prod_{\ell \neq k} \sin \left(\beta_{k}-\beta_{\ell}\right)}=\frac{\cos ^{m}(\alpha+z)}{\prod_{k=0}^{n} \sin \left(z-\beta_{k}\right)} .
\end{aligned}
$$

Two other summation formulae corresponding to Proposition 3 read as follows:

$$
\begin{aligned}
& \sum_{k=0}^{n} \frac{e^{i \beta_{k}} \sin ^{m}\left(\alpha+\beta_{k}\right)}{\prod_{\ell \neq k} \sin \left(\beta_{k}-\beta_{\ell}\right)}=e^{i(m \alpha+\mathcal{B})} \delta_{m, n}, \\
& \sum_{k=0}^{n} \frac{e^{i \beta_{k}} \cos ^{m}\left(\alpha+\beta_{k}\right)}{\prod_{\ell \neq k} \sin \left(\beta_{k}-\beta_{\ell}\right)}=i^{n} e^{i(m \alpha+\mathcal{B})} \delta_{m, n} .
\end{aligned}
$$

$\S 3.7$. Let $P(w)$ and $\mathcal{P}(w)$ be two trigonometric polynomials defined respectively by

$$
\begin{aligned}
& P\left(e^{i z}\right)=\sin ^{m}(\alpha+z) \quad \text { and } \quad P(w)=\frac{w^{-m}}{(2 i)^{m}} e^{m i \alpha}\left(w^{2}-e^{-2 i \alpha}\right)^{m}, \\
& \mathcal{P}\left(e^{i z}\right)=\cos ^{m}(\alpha+z) \quad \text { and } \quad \mathcal{P}(w)=\frac{w^{-m}}{2^{m}} e^{m i \alpha}\left(w^{2}+e^{-2 i \alpha}\right)^{m} .
\end{aligned}
$$

When $m$ and $n$ are two natural numbers with the different parity and $0 \leq m \leq n$, we derive from Theorem 4 the following two trigonometric sum identities:

$$
\begin{aligned}
& \sum_{k=0}^{n} \frac{e^{i\left(\beta_{k}-z\right)} \sin ^{m}\left(\alpha+\beta_{k}\right)}{\sin \left(z-\beta_{k}\right) \prod_{\ell \neq k} \sin \left(\beta_{k}-\beta_{\ell}\right)}=\frac{\sin ^{m}(\alpha+z)}{\prod_{k=0}^{n} \sin \left(z-\beta_{k}\right)}, \\
& \sum_{k=0}^{n} \frac{e^{i\left(\beta_{k}-z\right)} \cos ^{m}\left(\alpha+\beta_{k}\right)}{\sin \left(z-\beta_{k}\right) \prod_{\ell \neq k} \sin \left(\beta_{k}-\beta_{\ell}\right)}=\frac{\cos ^{m}(\alpha+z)}{\prod_{k=0}^{n} \sin \left(z-\beta_{k}\right)} .
\end{aligned}
$$


Their real and imaginary parts yield respectively the following identities:

$$
\begin{aligned}
& \sum_{k=0}^{n} \frac{\cot \left(z-\beta_{k}\right) \sin ^{m}\left(\alpha+\beta_{k}\right)}{\prod_{\ell \neq k} \sin \left(\beta_{k}-\beta_{\ell}\right)}=\frac{\sin ^{m}(\alpha+z)}{\prod_{k=0}^{n} \sin \left(z-\beta_{k}\right)}, \\
& \sum_{k=0}^{n} \frac{\cot \left(z-\beta_{k}\right) \cos ^{m}\left(\alpha+\beta_{k}\right)}{\prod_{\ell \neq k} \sin \left(\beta_{k}-\beta_{\ell}\right)}=\frac{\cos ^{m}(\alpha+z)}{\prod_{k=0}^{n} \sin \left(z-\beta_{k}\right)} ; \\
& \sum_{k=0}^{n} \frac{\sin ^{m}\left(\alpha+\beta_{k}\right)}{\prod_{\ell \neq k} \sin \left(\beta_{k}-\beta_{\ell}\right)}=\sum_{k=0}^{n} \frac{\cos ^{m}\left(\alpha+\beta_{k}\right)}{\prod_{\ell \neq k} \sin \left(\beta_{k}-\beta_{\ell}\right)}=0 .
\end{aligned}
$$

$\S 3.8$. When $P\left(e^{i z}\right)$ is a monomial of order $m$ in $\sin z$ and $\cos z$ with $0 \leq m \leq n$ and $m \equiv n(\bmod 2)$, there holds the following general identity:

$$
\sum_{k=0}^{n} \frac{P\left(e^{i \beta_{k}}\right)}{\sin \left(z-\beta_{k}\right) \prod_{j \neq k} \sin \left(\beta_{k}-\beta_{j}\right)}=\frac{P\left(e^{i z}\right)}{\prod_{k=0}^{n} \sin \left(z-\beta_{k}\right)} .
$$

For example, we have the following summation formulae:

$$
\begin{aligned}
& \sum_{k=0}^{n} \frac{\sin \left(\alpha+m \beta_{k}\right) \sin \left(\gamma+\ell \beta_{k}\right)}{\sin \left(z-\beta_{k}\right) \prod_{j \neq k} \sin \left(\beta_{k}-\beta_{j}\right)}=\frac{\sin (\alpha+m z) \sin (\gamma+\ell z)}{\prod_{k=0}^{n} \sin \left(z-\beta_{k}\right)}, \\
& \sum_{k=0}^{n} \frac{\sin \left(\alpha+m \beta_{k}\right) \cos \left(\gamma+\ell \beta_{k}\right)}{\sin \left(z-\beta_{k}\right) \prod_{j \neq k} \sin \left(\beta_{k}-\beta_{j}\right)}=\frac{\sin (\alpha+m z) \cos (\gamma+\ell z)}{\prod_{k=0}^{n} \sin \left(z-\beta_{k}\right)} \\
& \sum_{k=0}^{n} \frac{\cos \left(\alpha+m \beta_{k}\right) \cos \left(\gamma+\ell \beta_{k}\right)}{\sin \left(z-\beta_{k}\right) \prod_{j \neq k} \sin \left(\beta_{k}-\beta_{j}\right)}=\frac{\cos (\alpha+m z) \cos (\gamma+\ell z)}{\prod_{k=0}^{n} \sin \left(z-\beta_{k}\right)},
\end{aligned}
$$

where $\ell, m$, and $n$ are three integers satisfying $0 \leq|m|+|\ell| \leq n$ and $m+\ell \equiv n$ $(\bmod 2)$.

§3.9. When $P\left(e^{i z}\right)$ is a monomial of order $m$ in $\sin z$ and $\cos z$ with $0 \leq m \leq n$ and $m \not \equiv n(\bmod 2)$, there holds the following general identity:

$$
\sum_{k=0}^{n} \frac{e^{i\left(\beta_{k}-z\right)} P\left(e^{i \beta_{k}}\right)}{\sin \left(z-\beta_{k}\right) \prod_{j \neq k} \sin \left(\beta_{k}-\beta_{j}\right)}=\frac{P\left(e^{i z}\right)}{\prod_{k=0}^{n} \sin \left(z-\beta_{k}\right)}
$$

where the imaginary and real parts yield two summation formulae:

$$
\sum_{k=0}^{n} \frac{P\left(e^{i \beta_{k}}\right)}{\prod_{j \neq k} \sin \left(\beta_{k}-\beta_{j}\right)}=0 \quad \text { and } \quad \sum_{k=0}^{n} \frac{P\left(e^{i \beta_{k}}\right) \cot \left(z-\beta_{k}\right)}{\prod_{j \neq k} \sin \left(\beta_{k}-\beta_{j}\right)}=\frac{P\left(e^{i z}\right)}{\prod_{k=0}^{n} \sin \left(z-\beta_{k}\right)} .
$$

For example, we have the following summation formulae:

$$
\sum_{k=0}^{n} \frac{\sin ^{m}\left(\alpha+\beta_{k}\right) \sin ^{\ell}\left(\gamma+\beta_{k}\right)}{\prod_{j \neq k} \sin \left(\beta_{k}-\beta_{j}\right)}=\sum_{k=0}^{n} \frac{\sin ^{m}\left(\alpha+\beta_{k}\right) \cos ^{\ell}\left(\gamma+\beta_{k}\right)}{\prod_{j \neq k} \sin \left(\beta_{k}-\beta_{j}\right)}=\sum_{k=0}^{n} \frac{\cos ^{m}\left(\alpha+\beta_{k}\right) \cos ^{\ell}\left(\gamma+\beta_{k}\right)}{\prod_{j \neq k} \sin \left(\beta_{k}-\beta_{j}\right)}=0
$$


and

$$
\begin{aligned}
& \sum_{k=0}^{n} \cot \left(z-\beta_{k}\right) \frac{\sin ^{m}\left(\alpha+\beta_{k}\right) \sin ^{\ell}\left(\gamma+\beta_{k}\right)}{\prod_{j \neq k} \sin \left(\beta_{k}-\beta_{j}\right)}=\frac{\sin ^{m}(\alpha+z) \sin ^{\ell}(\gamma+z)}{\prod_{k=0}^{n} \sin \left(z-\beta_{k}\right)} \\
& \sum_{k=0}^{n} \cot \left(z-\beta_{k}\right) \frac{\sin ^{m}\left(\alpha+\beta_{k}\right) \cos ^{\ell}\left(\gamma+\beta_{k}\right)}{\prod_{j \neq k} \sin \left(\beta_{k}-\beta_{j}\right)}=\frac{\sin ^{m}(\alpha+z) \cos ^{\ell}(\gamma+z)}{\prod_{k=0}^{n} \sin \left(z-\beta_{k}\right)} \\
& \sum_{k=0}^{n} \cot \left(z-\beta_{k}\right) \frac{\cos ^{m}\left(\alpha+\beta_{k}\right) \cos ^{\ell}\left(\gamma+\beta_{k}\right)}{\prod_{j \neq k} \sin \left(\beta_{k}-\beta_{j}\right)}=\frac{\cos ^{m}(\alpha+z) \cos ^{\ell}(\gamma+z)}{\prod_{k=0}^{n} \sin \left(z-\beta_{k}\right)}
\end{aligned}
$$

where $\ell, m$, and $n$ are three integers satisfying $0 \leq|m|+|\ell| \leq n$ and $m+\ell \not \equiv n$ (mod 2). The examples exhibited here are far from exhausting.

There are other interesting trigonometric sum identities, for example, those appearing in Berndt [1], Chu [3], Chu-Marini [4], and Gessel [7]. The reader is encouraged to examine further.

\section{REFERENCES}

[1] B. C. Berndt, Explicit evaluations and reciprocity theorems for finite trigonometric sums Advances in Appl. Math. 29 (2002), 358-385. MR1942629 (2003h:11092)

[2] F. Calogero, Classical Many-Body Problems Amenable to Exact Treatments Lecture Notes in Physics: M66, Springer-Verlag (2001), Berlin. MR1880249 (2003a:37074)

[3] W. Chu, Summations on trigonometric functions Applied Mathematics and Computation 141 (2003), 161-176. MR1986078 (2004h:33003)

[4] W. Chu, A. Marini, Partial fractions and trigonometric identities Advances in Appl. Math. 23 (1999), 115-175. MR1699235 (2000i:33001)

[5] W. Chu, X. X. Wang, D. Y. Zheng, Application of the residue theorem to bilateral hypergeometric series Preprint, 2006.

[6] M. A. Dougall, On Vandermonde's theorem and some more general expansion Proc. Edinburgh. Math. Soc. 25 (1907), 114-132.

[7] I. M. Gessel, Generating functions and generalized Dedekind sums Elec. J. Combin. 4 (1997), \#R11. MR1444158 (98f:11032)

[8] R. A. Gustafson, Multilateral summation theorems for ordinary and basic hypergeometric series in $U(n)$ SIAM J. Math. Anal. 18 (1987), 1576-1596. MR911651 (90e:33015)

[9] R. A. Gustafson, Some q-beta and Mellin-Barnes integrals on compact Lie groups and Lie algebras Trans. Amer. Math. Soc. 341 (1994), 69-119. MR1139492 (94c:33032)

[10] R. Kress, Numerical Analysis: Graduate Texts in Mathematics (GTM181) Springer-Verlag (1998), Berlin-Heidelberg. MR1621952 (99c:65001)

[11] M. J. Mohlenkamp, L. Monzón, Trigonometric identities and sums of separable functions The Mathematical Intelligencer 27:2 (2005), 65-69. MR2156535

Department of Applied Mathematics, Dalian University of Technology, Dalian 116024, People's Republic of China

Current address: Dipartimento di Matematica, Università degli Studi di Lecce, Lecce-Arnesano, P. O. Box 193, 73100 Lecce, Italia

E-mail address: chu.wenchang@unile.it 